\title{
On a Semi-Smooth Newton Method and its Globalization
}

\author{
Kazufumi Ito $^{1}$ and Karl Kunisch ${ }^{2}$
}

May 21, 2007

\footnotetext{
${ }^{1}$ Center for Research in Scientific Computation, Department of Mathematics, North Carolina State University; research partially supported by the Army Research Office under DAAD19-02-1-039.

${ }^{2}$ Institut für Mathematik und Wissenschaftliches Rechnen, Universität Graz, Graz, Austria.
} 


\begin{abstract}
This paper addresses the globalization of the semi-smooth Newton method for non-smooth equations $F(x)=0$ in $\mathbb{R}^{m}$ with applications to complementarity and discretized $\ell^{1}$-regularization problems. Assuming semi-smoothness it is shown that super-linearly convergent Newton methods can be globalized, if appropriate descent directions are used for the merit function $|F(x)|^{2}$. Special attention is paid to directions obtained from the primal-dual active set strategy.
\end{abstract}




\section{Introduction}

We discuss the globalization of generalized Newton methods for non-smooth equations of the form $F(x)=0$ in $\mathbb{R}^{m}$. Examples which motivate our study include nonlinear variational inequalities and minimization of $\ell^{1}-$ functionals. Turning to nonlinear variational inequalities first we consider:

$$
\text { find } x \in \mathcal{C} \text { such that }
$$

$$
(f(x), y-x) \geq 0 \text { for all } y \in \mathcal{C},
$$

where $\mathcal{C}$ is a closed convex set in $\mathbb{R}^{m}$ and $f: \mathbb{R}^{m} \rightarrow \mathbb{R}^{m}$. This can equivalently be written as

$$
F(x)=x-\operatorname{Proj}_{\mathcal{C}}(x-f(x))=0,
$$

where $\operatorname{Proj}_{\mathcal{C}}$ is the projection of $\mathbb{R}^{m}$ onto $\mathcal{C}$. In particular, if $\mathcal{C}$ is a hypercube $\{x \mid \phi \leq x \leq \psi\}$ in $\mathbb{R}^{m}$, with $\phi \leq \psi$ and the inequalities are defined coordinatewise, then (1.2) can be equivalently expressed as

$$
0=F(x)=\left\{\begin{array}{l}
f(x)+\mu \\
\mu-\max (0, \mu+x-\psi)-\min (0, \mu+x-\phi),
\end{array}\right.
$$

where $\mu \in \mathbb{R}^{m}$ is the Lagrange multiplier. Similarly, the nonlinear complementarity system in $\mathbb{R}^{m}$

$$
f(x) \geq 0, \quad x \geq 0 \quad \text { and } \quad(f(x), x)=0
$$

can be equivalently written as

$$
F(x)=f(x)-\max (0, f(x)-x)=0 .
$$

Note that $F$ is a locally Lipschitz continuous function, if $f$ is locally Lipschitz, but it is not $C^{1}$, even if $f$ is $C^{1}$.

The other example of interest here involves the minimization of discretized $\ell^{1}$-functionals. For $\epsilon>0$ consider

$$
F(x)=f(x)+\frac{x-z}{\max (\epsilon,|x-z|)}=0,
$$

This equation arises, for example in robust estimation: find $x$ that minimizes

$$
U(x)+\sum_{i} \rho_{\epsilon}\left(x_{i}-z_{i}\right),
$$


where $U^{\prime}(x)=f(x)$ and

$$
\rho_{\epsilon}(s)= \begin{cases}|s| & \text { if }|s| \geq \epsilon \\ \frac{|x|^{2}}{2 \epsilon}+\frac{\epsilon}{2} & \text { if }|s| \leq \epsilon .\end{cases}
$$

The focus of this paper lies on globalization of generalized Newton methods based on the merit function

$$
\theta(x)=|F(x)|^{2}
$$

Newton methods are especially effective for stiff problems that may arise from the descretization of optimal control problem, free boundary problem and Black-Scholes tpye models in conjunction with American options. Here the Newton method provides a pre-conditioning of the gradient direction. We shall emphasize the construction and analysis of directions $d$ which act as descent directions for $\theta$ and which are at the same time quasi-Newton directions.

In Section 2 we establish sufficient conditions for a global convergence result. This result greatly benefits from earlier contributions in [FP, HPR, $\mathrm{P}, \mathrm{Q}, \mathrm{QS}]$. We shall subsequently consider two concepts of defining descent directions. The first are the primal-dual active set directions which were shown to be semi-smooth Newton directions, see [HIK, IK]. Secondly we shall consider traditional methods, like the Bouligand direction and generalized gradient directions, see e.g. $[\mathrm{P}, \mathrm{Q}, \mathrm{HPR}]$, which necessitate the solution of a non-smooth system to obtain the search direction $d$. To discuss the applicability of the global convergence result to these methods in a systematic manner we introduce, in Section 4, the notion of quasi-directional derivative.

As already mentioned we shall analyze in detail the primal-dual active set strategy, which is linked to semi-smooth Newton steps for max-type functions as in (1.5), (1.6). Our interest for globalizing the primal-dual active set strategy, based on the max-function as in (1.3) above, results from the fact that for the class of problems that we have in mind, the primal-dual active set strategy was extremely efficient, and required fewer iterations than the approach based on reformulations involving the Fischer-Burmeister functional, see e.g. [DK] and also [K2]. The class of problems that we are referring to are discretized optimal control problems for partial differential equations with e.g. control constraints, or variational inequalities, which can all be expressed in the form (1.5). Once the primal-dual active set directions are 
available it is only natural to also utilize them for globalization. The primaldual direction is constructed by solving a linear system that preserves certain properties of $f^{\prime}$, including possibly symmetry, positive definiteness and the $M$-property. Therefore, a part of the focus of this paper is put on the primal dual active set strategy. For problems of the type (1.5) it has the further advantage that the directions are obtained from a reduced system which needs to be solved on the currently inactive set only. For problems with bilateral constraints, (1.3) provides a very natural formulation of the complementary conditions. In the context of the Fischer-Burmeister approach a composition of the Fischer Burmeister functional can be used, which leads to more severe nonlinearities [K1]. In Section 3 we shall provide sufficient conditions of diagonal dominance type, which guarantee that the primal-dual active set direction satisfies the assumptions of Section 2 for global convergence. That is, there is no necessity to solve a nonlinear system and the same semi-smooth Newton-type direction can be taken for local as well as global steps. As a further observation on the comparison of utilizing the Fischer-Burmeister functional or the square of the max-function as in (1.7) for globalisation we recall that the former is $C^{1}$ while the latter is not, see e.g. [K2] and the references given there. As a consequence, in the Fisher Burmeister case, one can resort to the gradient of the merit function as a search direction, in case no element in the Bouligand subdifferential provides the necessary descent property. If $\theta$ is taken as merit function, one may have to solve a nonlinear equation to obtain a descent direction in this case. However, at points of non-differentiability of $\theta$ there are at most two elements in the Bouligand subdifferential of any coordinate of $F$, whereas the expression of the derivative of the square of the Fischer-Burmeister functional involves a Bouligand subdifferential whose cardinality is a continuum.

In Section 4 we systematically discuss alternative choices for descent direction which satisfy the conditions for global convergence of Section 2. These descent direction are obtained by means of solving a nonlinear equation. These restults are applied to bilaterally constrained problems in Section 4.1. In Section 4.2 the case of polyhedric constraints is considered.

In the remainder of this section we summarize differentiability concepts that will be utilized in the following sections and recall a local super-linear convergence result for semi-smooth Newton methods in finite dimensional spaces. The globalization strategy will be specified as well.

If $F$ is locally Lipschitz continuous on $\mathbb{R}^{m}$, then according to Rademacher's theorem, $F$ is differentiable almost everywhere. Let $D_{F}$ denote the set of 
points at which $F$ is differentiable and let $\partial_{B} F(x)$ be defined by

$$
\partial_{B} F(x)=\left\{J=\lim _{x_{i} \rightarrow x, x_{i} \in D_{F}} F^{\prime}\left(x_{i}\right)\right\} .
$$

We denote by $\partial F(x)$ the generalized derivative in the sense of Clarke, i.e.,

$$
\partial F(x)=\text { the convex hull of } \partial_{B} F(x) .
$$

A function $F$ is called directionally differentiable at $x \in \mathbb{R}^{m}$ if

$$
\lim _{t \rightarrow 0^{+}} \frac{F(x+t h)-F(x)}{t}=F^{\prime}(x ; h)
$$

exists for all $h \in \mathbb{R}^{m}$. $F$ is $\mathrm{B}$ (Bouligand)-differentiable $[\mathrm{R}]$ at $x$ if it is directionally differentiable at $x$ and

$$
\lim _{h \rightarrow 0} \frac{F(x+h)-F(x)-F^{\prime}(x ; h)}{|h|}=0 .
$$

It is shown in [S] that $F$ is B-differentiable at $x$ if and only if $F$ is directionally differentiable at $x$, provided that it is locally Lipschitz continuous at $x$.

The generalized Newton (or semi-smooth Newton) iterate is defined by

$$
x^{k+1}=x^{k}-V_{k}^{-1} F\left(x^{k}\right), \quad V_{k} \in \partial_{B} F\left(x^{k}\right),
$$

for $k=1,2, \ldots$ with $x^{0}$ given. Our particular interest in (1.10) is due to the fact that the primal-dual active set strategy for (1.4) was shown in [HIK] to be a specific semi-smooth Newton method in case $f$ is linear. The primal-dual active set strategy is known to be extremely efficient for solving discretized variational inequalities and constrained optimal control problems. The local convergence analysis for (1.10) relies on the concept of semi-smooth functions in $\mathbb{R}^{m}$, which we recall next:

Definition 1.1. $F: \mathbb{R}^{m} \rightarrow \mathbb{R}^{m}$ is called semismooth at $x$ if it is locally Lipschitz continuous at $x$ and

$$
\lim _{V \in \partial F\left(x+t h^{\prime}\right), h^{\prime} \rightarrow h, t \rightarrow 0^{+}} V h^{\prime} \text { exists for all } h \in \mathbb{R}^{m} .
$$


The notion of semi-smoothness was originally introduced for functionals by Miffin $[\mathrm{M}]$. Convex functions, smooth functions and subsmooth functions are examples for semi-smooth functions. Definition 1.1 is due to Qi and Sun. It was shown in [QS] that, if $F$ is semi-smooth at $x$, then the directional derivative of $F$ at $x$ exists in every direction $h$ and

$$
V h-F^{\prime}(x ; h)=o(|h|) \text { as } h \rightarrow 0,
$$

for all $V \in \partial F(x+h)$. Moreover if all $V \in \partial_{B} F(x)$ are nonsingular, then there exists a neighborhood $N(x)$ of $x$ and a constant $C$ such that for any $y \in N(x)$ and $V \in \partial_{B} F(y)$, we have $\left|V^{-1}\right| \leq C$. Combining these facts, the following result can be obtained, see e.g [Q].

Theorem 1.1. (Super-linear convergence) Suppose that $x^{*}$ is a solution to $F(x)=0$, that $F$ is semi-smooth at $x^{*}$, and all $V \in \partial_{B} F\left(x^{*}\right)$ are nonsingular. Then the iterates $\left\{x^{k}\right\}$ by (1.10) are well-defined and converge to $x^{*}$ super-linearly provided that $\left|x^{*}-x^{0}\right|$ is sufficiently small.

Throughout the remainder of this paper we assume that $F: \mathbb{R}^{m} \rightarrow$ $\mathbb{R}^{m}$ is locally Lipschitz continuous, directionally differentiable and that the following assumptions (A.1)-(A.3) hold.

- (A.1) $S=\left\{x \in \mathbb{R}^{m}:|F(x)| \leq\left|F\left(x^{0}\right)\right|\right\}$ is bounded.

- (A.2) There exist $\bar{\sigma}$ and $b>0$ such that for each $x \in S$ there exists $d=d(x) \in \mathbb{R}^{m}$ satisfying

$$
\theta^{\prime}(x ; d) \leq-\bar{\sigma} \theta(x) \quad \text { and } \quad|d| \leq b|F(x)| .
$$

- (A.3) The following closure property holds: if $x_{k} \rightarrow \bar{x}$ and $d\left(x_{k}\right) \rightarrow \bar{d}$ with $x_{k} \in S$, then $\theta^{\circ}(\bar{x} ; \bar{d}) \leq-\bar{\sigma} \theta(\bar{x})$.

It is assumed that $b>C$ is arbitrarily large parameter. It serves the purpose that the iterates $\left\{d_{k}\right\}$ can be uniformly bounded. For specific strategies for choosing $d_{k}$ bounds for $b$ are given in Section 3.1 and Section 4. See also Remark 2.1. (ii).

Here the Clarke generalized directional derivative $\theta^{\circ}(x ; d)[\mathrm{C}]$ of $\theta$ at $x$ in the direction $d$ is defined by

$$
\theta^{o}(x ; d)=\limsup _{y \rightarrow x, t \rightarrow 0^{+}} \frac{\theta(y+t d)-\theta(y)}{t} .
$$


Note that local Lipschitz continuity of $F$ implies Fréchet-differentiability of $\theta$ at $x^{*}$ if $F\left(x^{*}\right)=0$. Since $\theta^{\prime}(x ; d) \leq \theta^{o}(x ; d)$ for any $(x, d) \in \mathbb{R}^{m} \times \mathbb{R}^{m}$, and $\theta^{\prime}(x ; d(x)) \leq-\bar{\sigma} \theta(x)$ for $x \in S$, condition (A.3) is a strong closure property. Note that it is not assumed that $\bar{d}=\bar{d}(x)$.

We shall investigate the convergence properties of the following globalization algorithm to a solution $x^{*}$ of $F(x)=0$.

Algorithm Let $\beta, \gamma \in(0,1)$ and $\sigma \in(0, \bar{\sigma})$. Choose $x^{0} \in \mathbb{R}^{m}$ and set $k=0$. Given $x^{k}$ with $F\left(x^{k}\right) \neq 0$. Then:

(i) We select $V_{k} \in \partial_{B} F\left(x_{k}\right)$. If there exists a solution $h^{k}$ to

$$
V_{k} h^{k}=-F\left(x^{k}\right)
$$

with $\left|h^{k}\right| \leq b\left|F\left(x^{k}\right)\right|$, and satisfying

$$
\left|F\left(x^{k}+h^{k}\right)\right|<\gamma\left|F\left(x^{k}\right)\right|,
$$

then set $d^{k}=h^{k}, x^{k+1}=x^{k}+d^{k}, \alpha_{k}=1$, and $m_{k}=0$.

(ii) Otherwise choose $d^{k}=d\left(x^{k}\right)$ according to (A.2), - and in particular,

choose $d\left(x_{k}\right)=h_{k}$ from $(i)$, if $\theta^{\prime}\left(x^{k} ; h^{k}\right) \leq-\sigma \theta\left(x^{k}\right)$.

Then let $\alpha_{k}=\beta^{m_{k}}$, where $m_{k}$ is the first positive integer $m$ for which

$$
\theta\left(x^{k}+\beta^{m} d^{k}\right)-\theta\left(x^{k}\right) \leq-\sigma \beta^{m} \theta\left(x^{k}\right) .
$$

Set $x^{k+1}=x^{k}+\alpha_{k} d^{k}$.

In Section 3 we shall provide a sufficient condition for the two applications set forth in the introduction which guarantees that $d$ can always be chosen as the primal-dual active set direction in step (ii) of the Algorithm.

Condition (A.2) is motivated by the work in [HPR] where it is shown to guarantee a nontrivial step-size $\tau$ such that

$$
\theta(x+\tau d)-\theta(x) \leq-\sigma \tau \theta(x)
$$

if $\sigma \in(0, \bar{\sigma})$ and $x \in S$. We show that combined with (A.1) and (A.3) it also guarantees (subsequential) convergence of $\left\{x^{k}\right\}$ to a solution of $F(x)=0$. In $[\mathrm{FP}]$,Chapter 8 , and $[\mathrm{HPR}]$ variants of the condition

$$
\lim _{k \rightarrow \infty} F\left(x^{k}\right)^{T} F^{\prime}\left(x^{k} ; d^{k}\right) \geq \limsup _{k \rightarrow \infty} \frac{\theta\left(x^{k}+\tau^{k} d^{k}\right)-\theta\left(x^{k}\right)}{\tau^{k}}
$$

are used to analyze convergence of globalization schemes based on $\theta$ given by $|F(x)|^{2}$. Conditions (1.13) resembles (A.3) but the latter is simpler to check and more transparent also for the case that $F$ is $C^{1}$. 


\section{Semi-smooth Newton Method and Global- ization}

In this section we establish the main convergence theorem for the globalization algorithm presented in the introduction.

Theorem 2.1. Suppose that $F: \mathbb{R}^{m} \rightarrow \mathbb{R}^{m}$ is locally Lipschitz and Bdifferentiable.

(a) Assume that (A.1)-(A.3) hold. Then the sequence $\left\{x^{k}\right\}$ generated by the Algorithm is bounded, it satisfies $\left|F\left(x^{k+1}\right)\right|<\left|F\left(x^{k}\right)\right|$ for all $k \geq 0$, and each accumulation point $x^{*}$ of $\left\{x^{k}\right\}$ satisfies $F\left(x^{*}\right)=0$.

(b) If moreover for one such accumulation point

$$
|h| \leq c\left|F^{\prime}\left(x^{*} ; h\right)\right| \quad \text { for all } h \in \mathbb{R}^{m},
$$

then the sequence $x^{k}$ converges $x^{*}$.

(c) If in addition to the above assumptions $F$ is semi-smooth at $x^{*}$ and all $V \in \partial_{B} F\left(x^{*}\right)$ are nonsingular, then $x^{k}$ converges to $x^{*}$ superlinearly.

Proof. (a) First we prove that for each $x \in S$ such that $\theta(x) \neq 0$ and $d$ satisfying $\theta^{\prime}(x ; d) \leq-\bar{\sigma} \theta(x)$, there exists a $\bar{\tau}>0$ such that

$$
\theta(x+\tau d)-\theta(x) \leq-\sigma \tau \theta(x) \text { for all } \tau \in[0, \bar{\tau}] .
$$

If this is not the case, then there exits a sequence $\tau_{n} \rightarrow 0^{+}$such that

$$
\theta\left(x+\tau_{n} d\right)-\theta(x)>-\sigma \tau_{n} \theta(x) .
$$

Dividing both sides by $\tau_{n}$ and letting $n \rightarrow \infty$, we have by (1.12) of (A.2) that

$$
-\bar{\sigma} \theta(x) \geq \theta^{\prime}(x ; d) \geq-\sigma \theta(x) .
$$

Since $\sigma<\bar{\sigma}$, this shows $\theta(x)=0$, which contradicts the assumption $\theta(x) \neq 0$. Hence for each level $k$ at which $d^{k}=d\left(x^{k}\right)$ is chosen according to the second alternative in the Algorithm there exists $m^{k}<\infty$ and $\alpha_{k}>0$ such that $\left|F\left(x^{k+1}\right)\right|<\left|F\left(x^{k}\right)\right|$. By construction the iterates therefore satisfy $\left|F\left(x^{k+1}\right)\right|<\left|F\left(x^{k}\right)\right|$ for each $k \geq 0$.

Assume first that $\lim \sup \alpha_{k}>0$. If the first alternative in the Algorithm with $\alpha_{k}=1$ occurs infinitely many times then, using the fact that $\gamma<1$, we find that $\lim _{k \rightarrow 0} \theta\left(x^{k}\right)=0$. Otherwise, for all $k$ sufficiently large

$$
0 \leq \theta\left(x^{k+1}\right) \leq\left(1-\sigma \alpha_{k}\right) \theta\left(x^{k}\right) \leq \theta\left(x^{k}\right) .
$$


Thus $\theta\left(x^{k}\right)$ is monotonically decreasing, bounded below by 0 and hence convergent. Therefore $\lim _{k \rightarrow \infty}\left(\theta\left(x^{k+1}\right)-\theta\left(x^{k}\right)\right)=0$ and consequently $\lim _{k \rightarrow \infty} \alpha_{k} \theta\left(x^{k}\right)=$ 0 . Thus $\lim \sup \alpha_{k}>0$ implies that $\lim _{k \rightarrow \infty} \theta\left(x^{k}\right)=0$. Hence each accumulation point $x^{*}$ of $\left\{x^{k}\right\}$ satisfies $F\left(x^{*}\right)=0$. The existence of an accumulation point follows from (A.1).

If on the other hand $\lim \sup \alpha_{k}=0$, then $\lim m_{k} \rightarrow \infty$. By the definition of $m_{k}$, for $\tau_{k}:=\beta^{m_{k}-1}$, we have $\tau_{k} \rightarrow 0$ and

$$
\theta\left(x^{k}+\tau_{k} d^{k}\right)-\theta\left(x^{k}\right)>-\sigma \tau_{k} \theta\left(x^{k}\right) .
$$

By (A.1), (A.2) the sequence $\left\{\left(x^{k}, d^{k}\right)\right\}$ is bounded. Let $\left\{\left(x^{k}, d^{k}\right)\right\}_{k \in K}$ be any convergent subsequence with limit $\left(x^{*}, d\right)$. Note that

$$
\frac{\theta\left(x^{k}+\tau_{k} d^{k}\right)-\theta\left(x^{k}\right)}{\tau_{k}}=\frac{\theta\left(x^{k}+\tau_{k} d\right)-\theta\left(x^{k}\right)}{\tau_{k}}+\frac{\theta\left(x^{k}+\tau_{k} d^{k}\right)-\theta\left(x^{k}+\tau_{k} d\right)}{\tau_{k}},
$$

where

$$
\lim _{k \in K, k \rightarrow \infty} \frac{\theta\left(x^{k}+\tau_{k} d^{k}\right)-\theta\left(x^{k}+\tau_{k} d\right)}{\tau_{k}} \rightarrow 0,
$$

since $\theta$ is locally Lipschitz continuous. From (2.2) and (A.3) we find

$$
-\sigma \theta\left(x^{*}\right) \leq \limsup _{k \in K, k \rightarrow \infty} \frac{\theta\left(x^{k}+\tau_{k} d^{k}\right)-\theta\left(x^{k}\right)}{\tau_{k}} \leq \theta^{o}\left(x^{*} ; d\right) \leq-\bar{\sigma} \theta\left(x^{*}\right) .
$$

It follows that $(\bar{\sigma}-\sigma) \theta\left(x^{*}\right) \leq 0$ and thus $\theta\left(x^{*}\right)=0$.

(b) Since $F$ is B-differentiable at $x^{*}$ there exists a $\delta>0$ such that for $\left|x-x^{*}\right| \leq$ $\delta$

$$
\left|F(x)-F\left(x^{*}\right)-F^{\prime}\left(x^{*} ; x-x^{*}\right)\right| \leq \frac{1}{2 c}\left|x-x^{*}\right| .
$$

Thus,

$$
\begin{aligned}
& \left|F^{\prime}\left(x^{*} ; x-x^{*}\right)\right| \leq|F(x)|+\left|F(x)-F\left(x^{*}\right)-F^{\prime}\left(x^{*}, x-x^{*}\right)\right| \\
& \quad \leq|F(x)|+\frac{1}{2 c}\left|x-x^{*}\right| .
\end{aligned}
$$

From (2.1)

$$
\left|x-x^{*}\right| \leq c\left|F^{\prime}\left(x^{*} ; x-x^{*}\right)\right| \leq c|F(x)|+\frac{1}{2}\left|x-x^{*}\right|
$$


and thus

$$
\left|x-x^{*}\right| \leq 2 c|F(x)|, \quad \text { if }\left|x-x^{*}\right| \leq \delta .
$$

Given $\epsilon \in(0, \delta)$ define the set

$$
N\left(x^{*}, \epsilon\right)=\left\{x \in \mathbb{R}^{m}:\left|x-x^{*}\right| \leq \epsilon, \quad|F(x)| \leq \frac{\epsilon}{2 c+b}\right\} .
$$

Since $x^{*}$ is an accumulation point of $\left\{x^{k}\right\}$, there exists an index $\bar{k}$ such that $x^{\bar{k}} \in N\left(x^{*}, \epsilon\right)$. Note that $\left|d^{k}\right| \leq b\left|F\left(x^{k}\right)\right|$ for all $k$. In case the direction is chosen according to (i) of the Algorithm this follows from the assumption that $C<b$, and it follows from (1.12) if the direction is chosen according to (ii).

It therefore follows that

$$
\begin{gathered}
\left|x^{\bar{k}+1}-x^{*}\right| \leq\left|x^{\bar{k}}-x^{*}+\alpha_{\bar{k}} d^{\bar{k}}\right| \leq\left|x^{\bar{k}}-x^{*}\right|+\alpha_{\bar{k}}\left|d^{\bar{k}}\right| \\
\leq 2 c\left|F\left(x^{\bar{k}}\right)\right|+b\left|F\left(x^{\bar{k}}\right)\right|=(2 c+b)\left|F\left(x^{\bar{k}}\right)\right| \leq \epsilon .
\end{gathered}
$$

Hence $x^{\bar{k}+1} \in N\left(x^{*}, \epsilon\right)$. By induction, $x^{k} \in N\left(x^{*}, \epsilon\right)$ for all $k \geq \bar{k}$ and thus the sequence $x^{k}$ converges to $x^{*}$.

(c) Since $\lim _{k \rightarrow \infty} x^{k}=x^{*}$ the iterates $x^{k}$ of the Algorithm enter into the region of attraction for Theorem 1.1. By Lemma 2.1 below with $x=x^{k}$, there exists for any $\gamma \in(0,1)$ a constant $k_{\gamma}$ such that the iterates according to (1.10) satisfy $\left|F\left(x^{k+1}\right)\right| \leq \gamma\left|F\left(x^{k}\right)\right|$, for $k \geq k_{\gamma}$. Hence these iterates coincide with those of the Algorithm for $k \geq k_{\gamma}$, and super-linear convergence follows.

Lemma 2.1. Suppose that $F: \mathbb{R}^{m} \rightarrow \mathbb{R}^{m}$ is semi-smooth at a solution $x^{*}$ of $F(x)=0$, and that all $V \in \partial_{B} F\left(x^{*}\right)$ are nonsingular. Then there exist a neighborhood $N$ of $x^{*}$, a constant $C$ and $\epsilon: \mathbb{R}^{+} \rightarrow \mathbb{R}^{+}$with $\lim _{t \rightarrow 0^{+}} \epsilon(t)=0$ such that $\left|V^{-1}(x)\right| \leq C$ for all $x \in N$ and

$$
\begin{aligned}
& \left|x-V^{-1} F(x)-x^{*}\right| \leq \epsilon\left(\left|x-x^{*}\right|\right)\left|x-x^{*}\right| \\
& \left|F\left(x-V^{-1} F(x)\right)\right| \leq \epsilon\left(\left|x-x^{*}\right|\right)|F(x)|,
\end{aligned}
$$

for all $V \in \partial_{B} F(x)$ and $x \in N$.

For the proof we refer to $[\mathrm{Q}]$. 
Remark 2.1. (i) We point out that the closure property (A.3) is used in the proof of Theorem 2.1 only for the case that $\lim \sup _{k \rightarrow \infty} \alpha_{k}=0$.

(ii) For part (a) of Theorem 2.1 the condition $\left|h^{k}\right| \leq b\left|F\left(x^{k}\right)\right|$ in the first alternative of the Algorithm is not required and $|d| \leq b|F(x)|$ for all $x \in S$ used in alternative (ii) can be replaced by requiring that the directions are uniformly bounded, or that $\left|d^{k}\right| \leq b\left|F\left(x^{k}\right)\right|$ for all $k=1,2, \ldots$ These conditions are used in the proof of Theorem 2.1(b).

(iii) Since $h \rightarrow F^{\prime}\left(x^{*} ; h\right)$ is positively homogeneous one can easily argue that (2.1) is equivalent to the condition that $F^{\prime}\left(x^{*} ; h\right)=0$ implies that $h=0$.

\section{Primal-Dual Direction}

In this section we provide sufficient conditions so that the semi-smooth Newton direction given by the primal-dual active set strategy can also be used as a descent direction for the globalization. The essential assumption requires diagonal dominance of $f$ in (1.3), in a sense to be specified in (3.7) below.

\subsection{Box Constraints}

We return to the example (1.1) which was already presented in the Introduction, with $\mathcal{C}$ chosen as box constraints. It arises for instance from the variational problem:

$$
\min \Phi(x) \text { subject to } x \in \mathcal{C},
$$

where $\mathcal{C}=\{x \mid \phi \leq x \leq \psi\}$. In this case $f(x)=\Phi^{\prime}(x)$. Unilateral constraints are included as special cases with $\phi=-\infty$ or $\psi=\infty$. As discussed before, this variational problem results in (1.3), where we assume that $f$ is $C^{1}$. In (1.3) we can eliminate $\mu$ by $\mu=-f(x)$ and work with the single variable $x$. This leads to the equation

$$
F(x)=f(x)+\max (0,-f(x)+c(x-\psi))+\min (0,-f(x)+c(x-\phi))=0,
$$

which is equivalent to (1.3) for any $c>0$. Define

$$
\begin{array}{ll}
\mathcal{A}^{+}=\{-f(x)+c(x-\psi)>0\}, & \mathcal{A}^{-}=\{-f(x)+c(x-\phi)<0\} \\
\mathcal{I}^{1}=\{-f(x)+c(x-\psi)=0\}, & \mathcal{I}^{2}=\{c(x-\psi)<f(x)<c(x-\phi)\} \quad \text { and } \\
\mathcal{I}^{3}=\{-f(x)+c(x-\phi)=0\}, &
\end{array}
$$


where $\mathcal{A}^{+}=\{-f(x)+c(x-\psi)>0\}$ stands for $\left\{i:\left(-f(x)_{i}+c(x-\psi)_{i}>0\right\}\right.$, and analogously for the other sets. We obtain

$$
F^{\prime}(x ; d)= \begin{cases}c d & \text { on } \mathcal{A}^{+} \cup \mathcal{A}^{-} \\ f^{\prime}(x) d & \text { on } \mathcal{I}^{2} \\ \max \left(f^{\prime}(x) d, c d\right) & \text { on } \mathcal{I}^{1} \\ \min \left(f^{\prime}(x) d, c d\right) & \text { on } \mathcal{I}^{3}\end{cases}
$$

and

$$
\begin{gathered}
\frac{1}{2} \theta^{\prime}(x ; d)=(c(x-\psi), c d)_{\mathcal{A}^{+}}+(c(x-\phi), c d)_{\mathcal{A}^{-}}+\left(f(x), f^{\prime}(x) d\right)_{\mathcal{I}^{2}} \\
+\left(f(x), \max \left(f^{\prime}(x) d, c d\right)\right)_{\mathcal{I}^{1}}+\left(f(x), \min \left(f^{\prime}(x) d, c d\right)\right)_{\mathcal{I}^{3}}
\end{gathered}
$$

Here 'on a set' means 'for all indices in the set', for example, $\left(F^{\prime}(x ; d)\right)_{i}=$ $\min \left(\left(f^{\prime}(x) d\right)_{i}, c d_{i}\right)$ for $i \in \mathcal{I}^{3}$.

The primal-dual active set strategy was found to be very effective for box constrained optimization problems both in finite $[\mathrm{KR}]$ and in infinite dimensional problems, see e.g. [HIK, IK], and its local convergence properties were analyzed in these papers. Here we discuss the feasibility of utilizing this Newton step given by the primal-dual strategy also for globalization. According to the Algorithm of Section 1 we propose to take a full step, if the step $h_{k}$ determined by this strategy satisfies the descent property $\mid F\left(x^{k}+\right.$ $\left.h^{k}\right)|\leq \gamma| F\left(x^{k}\right) \mid$. Otherwise we check whether it can serve as a descent direction. Below we shall provide a sufficient condition for this to be the case in the sense that (A.2) holds and we shall also verify the closure property (A.3).

For the primal-dual active set method the steps $d^{k}$ in (i) and (ii) of the Algorithm are determined according to

$$
\left\{\begin{array}{l}
d^{k}=y^{k+1}-x^{k} \\
f^{\prime}\left(x^{k}\right)\left(y^{k+1}-x^{k}\right)+f\left(x^{k}\right)=0 \text { in } \mathcal{I}_{k} \\
y^{k+1}=\psi \text { on } \mathcal{A}_{k}^{+}, \quad x^{k+1}=\phi \text { on } \mathcal{A}_{k}^{-}
\end{array}\right.
$$


where the index sets $\mathcal{A}_{k}^{+}, \mathcal{A}_{k}^{-}, \mathcal{I}_{k}$ are defined by

$$
\begin{aligned}
& \mathcal{A}_{k}^{+}=\left\{\left(-f\left(x^{k}\right)_{i}+c\left(x^{k}-\psi\right)_{i}>0\right\},\right. \\
& \mathcal{A}_{k}^{-}=\left\{\left(-f\left(x^{k}\right)_{i}+c\left(x^{k}-\phi\right)_{i}<0\right\}, \quad \mathcal{I}_{k}=\left(\mathcal{A}_{k}^{+} \cup \mathcal{A}_{k}^{-}\right)^{c},\right.
\end{aligned}
$$

and 'on $\mathcal{A}_{k}^{+}$' stands for 'for all $i \in \mathcal{A}_{k}^{+}$, and analogously for $\mathcal{A}_{k}^{-}$and $\mathcal{I}_{k}$. In the case of no constraints, the resulting iteration coincides with the Newton iteration, see e.g. [OR]. Here we must show clarify that this is a semi-smooth Newton step in the sense of (1.10). This amounts to showing that

$$
V(x) d=\left\{\begin{array}{l}
f^{\prime}(x) d \text { on } \mathcal{I}^{1} \cup \mathcal{I}^{2} \cup \mathcal{I}^{3}, \\
c d \text { on } \mathcal{A}^{+} \cup \mathcal{A}^{-}
\end{array}\right.
$$

is an element of $\partial_{B} F(x)$, for $x \in S$. Note that for the scalar max-function we have

$$
\partial_{B} \max (0, x)=\left\{\begin{array}{cc}
0 & \text { for } x<0 \\
\{0,1\} & \text { for } x=0 \\
1 & \text { for } x>0
\end{array}\right.
$$

and the choice in (3.3) corresponds to choosing 0 as the value in $\partial_{B} \max (0, x)$ if $x=0$. To distinguish between the scalar- and the vector-valued max operation we introduce

$$
\partial_{b} F(x)=\otimes_{i=1}^{m} \partial_{B} F_{i}(x),
$$

where $F_{i}$ is the i-th coordinate of $F$. From the definition of $\partial_{B} F(x)$ it follows that $\partial_{B} F(x) \subset \partial_{b} F(x)$. A sufficient condition for the converse inclusion to hold is that $c I-f^{\prime}(x)$ is nonsingular, which we suppose to hold for all $x \in S$. Thus $d^{k}$ satisfies $V\left(x^{k}\right) d^{k}+F\left(x^{k}\right)=0$ with $V\left(x^{k}\right) \in \partial_{B} F\left(x^{k}\right)$.

We henceforth set

$$
\mathcal{A}_{k}=\mathcal{A}_{k}^{+} \cup \mathcal{A}_{k}^{-}
$$

We have

$$
\begin{aligned}
& \frac{1}{2} \theta^{\prime}\left(x^{k} ; d^{k}\right)=-c^{2}\left|d^{k}\right|_{\mathcal{A}_{k}}^{2}-\left|f^{\prime}\left(x^{k}\right) d^{k}\right|_{\mathcal{I}_{k}^{2}}^{2} \\
& \quad+\left(f\left(x^{k}\right), \max \left(f^{\prime}\left(x^{k}\right) d^{k}, c d^{k}\right)\right)_{\mathcal{I}_{k}^{1}}+\left(f\left(x^{k}\right), \min \left(f^{\prime}\left(x^{k}\right) d^{k}, c d^{k}\right)\right)_{\mathcal{I}_{k}^{3}},
\end{aligned}
$$


where $\mathcal{I}_{k}^{i}$ is defined as $\mathcal{I}^{i}$ with $x$ replaced by $x^{k}$. Note that by (3.2)

$$
\begin{gathered}
\left(f\left(x^{k}\right), \max \left(f^{\prime}\left(x^{k}\right) d^{k}, c d^{k}\right)\right)_{\mathcal{I}_{k}^{1}}=-\left(f^{\prime}\left(x^{k}\right) d^{k}, \max \left(f^{\prime}\left(x^{k}\right) d^{k}, c d^{k}\right)\right) \\
=\left\{\begin{array}{l}
-\left|f^{\prime}\left(x^{k}\right) d^{k}\right|^{2} \text { on } \mathcal{I}_{k}^{11} \\
-\left(f^{\prime}\left(x^{k}\right) d^{k}, c d^{k}\right) \text { on } \mathcal{I}_{k}^{12}
\end{array}\right.
\end{gathered}
$$

and similarly,

$$
\begin{gathered}
\left(f\left(x^{k}\right), \max \left(f^{\prime}\left(x^{k}\right) d^{k}, c d^{k}\right)\right)_{\mathcal{I}_{k}^{3}}=-\left(f^{\prime}\left(x^{k}\right) d^{k}, \min \left(f^{\prime}\left(x^{k}\right) d^{k}, c d^{k}\right)\right) \\
=\left\{\begin{array}{l}
-\left|f^{\prime}\left(x^{k}\right) d^{k}\right|^{2} \text { on } \mathcal{I}_{k}^{31} \\
-\left(f^{\prime}\left(x^{k}\right) d^{k}, c d^{k}\right) \text { on } \mathcal{I}_{k}^{32},
\end{array}\right.
\end{gathered}
$$

where

$$
\mathcal{I}^{1}=\mathcal{I}^{11} \cup \mathcal{I}^{12}=\left\{i \in \mathcal{I}^{1}:\left(f^{\prime}(x) d\right)_{i} \geq c d_{i}\right\} \cup\left\{i \in \mathcal{I}^{1}:\left(f^{\prime}(x) d\right)_{i}<c d_{i}\right\}
$$

and

$$
\mathcal{I}^{3}=\mathcal{I}^{31} \cup \mathcal{I}^{32}=\left\{i \in \mathcal{I}^{3}:\left(f^{\prime}(x) d\right)_{i} \leq c d_{i}\right\} \cup\left\{i \in \mathcal{I}^{3}:\left(f^{\prime}(x) d\right)_{i}>c d_{i}\right\},
$$

and the subscript $k$ denotes evaluation at $x^{k}$. Thus, setting $\mathcal{I I}_{k}=\mathcal{I}_{k}^{2} \cup \mathcal{I}_{k}^{11} \cup$ $\mathcal{I}_{k}^{31}$ and $\mathcal{I} \mathcal{A}_{k}=\mathcal{I}_{k}^{12} \cup \mathcal{I}_{k}^{32}$, then

$$
\frac{1}{2} \theta^{\prime}\left(x^{k} ; d^{k}\right)=-c^{2}\left|d^{k}\right|_{\mathcal{A}_{k}}^{2}-\left|f^{\prime}\left(x^{k}\right) d^{k}\right|_{\mathcal{I I}_{k}}^{2}-c\left(f^{\prime}\left(x^{k}\right) d^{k}, d^{k}\right)_{\mathcal{I} \mathcal{A}_{k}} .
$$

We shall return to this expression to verify (A.2). Before that we turn to (A.3) and assume that $\left(x^{k}, d^{k}\right) \rightarrow(\bar{x}, \bar{d})$ and $V\left(x^{k}\right) d^{k}+F\left(x^{k}\right)=0$. Then, $(\bar{x}, \bar{d})$ satisfies

$$
\begin{aligned}
& \bar{d}+c(\bar{x}-\psi)=0 \text { on } \mathcal{A}^{+}=\{-f(\bar{x})+c(\bar{x}-\psi)>0\} \\
& \bar{d}+c(\bar{x}-\phi)=0 \text { on } \mathcal{A}^{-}=\{-f(\bar{x})+c(\bar{x}-\phi)<0\} \\
& f^{\prime}(\bar{x}) \bar{d}+f(\bar{x})=0 \text { on } \mathcal{I}^{2}=\{c(\bar{x}-\phi)<f(\bar{x})<c(\bar{x}-\psi)\} \\
& f^{\prime}(\bar{x}) \bar{d}+f(\bar{x})=0 \text { or } c \bar{d}+f(\bar{x})=0 \text { on }\left\{\begin{array}{l}
\mathcal{I}^{1}=\{-f(\bar{x})+c(\bar{x}-\psi)=0\} \\
\text { and } \\
\mathcal{I}^{3}=\{-f(\bar{x})+c(\bar{x}-\phi)=0\}
\end{array}\right.
\end{aligned}
$$


The properties of the last line follow from the fact that on $\mathcal{I}^{1} \cup \mathcal{I}^{3}$ we have that if $x^{k} \in \mathcal{I}_{k}$ for infinitely many $k$, then $f^{\prime}(\bar{x}) \bar{d}+F(\bar{x})=0$ and if $x^{k} \in \mathcal{A}_{k}$ for infinitely many $k$, then $c \bar{d}+F(\bar{x})=0$. Note that

$$
F(\bar{x})=\left\{\begin{array}{l}
f(\bar{x}) \text { on } \mathcal{I}=\mathcal{I}^{1} \cup \mathcal{I}^{2} \cup \mathcal{I}^{3} \\
c(\bar{x}-\psi) \text { on } \mathcal{A}^{+} \\
c(\bar{x}-\phi) \text { on } \mathcal{A}^{-} .
\end{array}\right.
$$

Next, it can be shown (see, Section 4) that

$$
\theta^{0}(\bar{x}, \bar{d})=2\left(F(\bar{x}), F^{0}(\bar{x}, \bar{d})\right)
$$

where

$$
F^{0}(\bar{x}, \bar{d})=F^{\prime}(\bar{x}, \bar{d}) \text { except on the set } \mathcal{I}^{1} \cup \mathcal{I}^{3}
$$

and on $\mathcal{I}^{1} \cup \mathcal{I}^{3}$ we have

$$
\begin{aligned}
& F^{0}(\bar{x}, \bar{d})=\max \left(f^{\prime}(\bar{x}) \bar{d}, c \bar{d}\right) \text { on }\{F(\bar{x})>0\} \\
& F^{0}(\bar{x}, \bar{d})=\min \left(f^{\prime}(\bar{x}) \bar{d}, c \bar{d}\right) \text { on }\{F(\bar{x}) \leq 0\} .
\end{aligned}
$$

Thus, we find

$$
\left(F(\bar{x}), F^{o}(\bar{x}, \bar{d})\right)_{\mathcal{I}^{1} \cup \mathcal{I}^{3}}=\left\{\begin{array}{l}
-\left|f^{\prime}(\bar{x}) \bar{d}\right|^{2} \text { on } \mathcal{J}^{1} \\
-c^{2}|\bar{d}|^{2} \text { on } \mathcal{J}^{2} \\
-c\left(f^{\prime}(\bar{x}) \bar{d}, \bar{d}\right) \text { on } \mathcal{J}^{3}
\end{array}\right.
$$

where

$$
\begin{aligned}
\mathcal{J}^{1} & \left.=\left\{i \in \mathcal{I}^{1} \cup \mathcal{I}^{3}: F(\bar{x})_{i}=-\left(f^{\prime}(\bar{x}) \bar{d}\right)_{i} \text { and } F(\bar{x})_{i}\left(f^{\prime}(\bar{x}) \bar{d}\right)_{i}-\bar{c} d_{i}\right)>0\right\} \\
\mathcal{J}^{2} & \left.=\left\{i \in \mathcal{I}^{1} \cup \mathcal{I}^{3}: F(\bar{x})_{i}=-c \bar{d}_{i} \text { and } F(\bar{x})_{i}\left(f^{\prime}(\bar{x}) \bar{d}\right)_{i}-c \bar{d}_{i}\right)<0\right\} \\
\mathcal{J}^{3} & =\mathcal{I}^{1} \cup \mathcal{I}^{3} \backslash\left(\mathcal{J}^{1} \cup \mathcal{J}^{2}\right),
\end{aligned}
$$

Thus, with $\mathcal{A} \mathcal{A}=\mathcal{A}^{+} \cup \mathcal{A}^{-} \cup \mathcal{J}^{2}, \mathcal{I} \mathcal{I}=\mathcal{I}^{2} \cup \mathcal{J}^{1}$ and $\mathcal{I} \mathcal{A}=\mathcal{J}^{3}$, we have

$$
\theta^{o}(\bar{x} ; \bar{d})=-c^{2}|\bar{d}|_{\mathcal{A} \mathcal{A}}^{2}-\left|f^{\prime}(\bar{x}) \bar{d}\right|_{\mathcal{I} \mathcal{I}}^{2}-c\left(f^{\prime}(\bar{x}) \bar{d}, \bar{d}\right)_{\mathcal{I} \mathcal{A}} .
$$

Now we consider (A.2) and (A.3) together, and let $A=f^{\prime}(x), x \in S$ and $\mathcal{A A} \cup \mathcal{I I} \cup \mathcal{I} \mathcal{A}$ be any arbitrary disjoint partition of the index set $1, \ldots, n$ and set

$$
A=\left(\begin{array}{ccc}
A_{11} & A_{12} & A_{13} \\
A_{21} & A_{22} & A_{23} \\
A_{31} & A_{32} & A_{33}
\end{array}\right)
$$


where without loss of generality we rearranged the indices according to the partition, with $1,2,3$ corresponding to $\mathcal{A} \mathcal{A}, \mathcal{I} \mathcal{I}, \mathcal{I} \mathcal{A}$, respectively. From (3.4) and (3.6) we must consider the quadratic form for $d=\left(d_{1}, d_{2}, d_{3}\right)$

$Q(d, d)=c^{2}\left|d_{1}\right|_{1}^{2}+\left|(A d)_{2}\right|_{2}^{2}+c\left((A d)_{3}, d_{3}\right)_{3}=c^{2}\left|d_{1}\right|_{1}^{2}+\left|f_{2}\right|_{2}^{2}+c\left(A_{31} d_{1}+A_{32} d_{2}+A_{33} d_{3}, d_{3}\right)_{3}$,

where

$$
f=A_{21} d_{1}+A_{22} d_{2}+A_{23} d_{3}
$$

Thus,

$$
d_{2}=A_{22}^{-1}\left(f-A_{21} d_{1}-A_{23} d_{3}\right)
$$

and

$$
\begin{gathered}
Q(d, d)=c^{2}\left|d_{1}\right|_{1}^{2}+\left|f_{2}\right|_{2}^{2}+c\left(\left(A_{31}-A_{32} A_{22}^{-1} A_{21}\right) d_{1}+A_{32} A_{22}^{-1} f_{2}, d_{3}\right)_{3} \\
+c\left(\left(A_{33}-A_{32} A_{22}^{-1} A_{23}\right) d_{3}, d_{3}\right)_{3},
\end{gathered}
$$

where $Q$ depends on $x \in S$. We assume that

$$
\left\{\begin{array}{l}
c\left(A_{33}-A_{32} A_{22}^{-1} A_{23}\right) \\
-\frac{1}{4}\left(\left(A_{31}-A_{32} A_{22}^{-1} A_{21}\right)^{T}\left(A_{31}-A_{32} A_{22}^{-1} A_{21}\right)+c^{2}\left(A_{32} A_{22}^{-1}\right)^{T}\left(A_{32} A_{22}^{-1}\right)\right) \\
\text { is positive definite for arbitrary } x \in S \text { and arbitrary partitions } \mathcal{A} \mathcal{A}, \mathcal{I} \mathcal{I}, \mathcal{I} \mathcal{A} .
\end{array}\right.
$$

Condition (3.7) requires a diagonal dominance of $A$, as will further be discussed in Remark 3.1. below. Using $a^{2}+\frac{b^{2}}{4}-a b \geq 0$, this condition implies that $Q$ is positive definite for every $x \in S$, and, since $S$ is compact if (A.1) holds, $Q$ is also uniformly positive definite for $x \in S$. In the case without constraints, i.e. $\psi=\infty$ and $\phi=-\infty$, or when the sets $\mathcal{I}^{1}$ and $\mathcal{I}^{3}$ are empty, the subsets $\mathcal{I} \mathcal{A}$ are empty, and (3.7) can be considered to be vacuously satisfied. In case of unilateral constraints, e.g. with $\phi=-\infty$, the sets $\mathcal{I}^{3}$ and consequently $\mathcal{I}^{3 j}, j=1,2,3$ are empty, and $\mathcal{I} \mathcal{A}=\mathcal{I}^{13}$ only.

If strict complementarity holds, in the sense that $\left(x^{*}\right)_{i}=\psi_{i}$ implies that $\lambda_{i}^{*}=-f\left(x^{*}\right)_{i}>0$ and $\left(x^{*}\right)_{i}=\phi_{i}$ implies that $\lambda_{i}^{*}=-f\left(x^{*}\right)_{i}<0$, then $\mathcal{I}^{1}$ and $\mathcal{I}^{3}$ are empty at $x^{*}$ and hence these sets are also empty for all $x$ in a neighborhood of $x^{*}$. Consequently the sets $\mathcal{I} \mathcal{A}$ are empty and (3.7) is satisfied locally in a neighborhood of $x^{*}$. 
Henceforth let $(x, d)$ be either a step $\left(x^{k}, d^{k}\right)$ determined by the active set strategy or a limit point $(\bar{x}, \bar{d})$ as in (A.3). Then by (3.4), (3.6), (3.7) there exists $\hat{\sigma}$ such that

$$
\theta^{\prime}(x ; d) \leq-\hat{\sigma}|d|^{2} \quad \text { and } \quad \theta^{o}(x ; d) \leq-\hat{\sigma}|d|^{2},
$$

independently of such $(x, d)$.

Moreover, if $(x, d)$ is a step from the active set strategy we have

$$
A d=F(x) \text { on } \mathcal{A} \text {, and } d=F(x) \text { on } \mathcal{I},
$$

where $A=f^{\prime}(x)$. Consequently

$$
\left|F(x)_{\mathcal{I}(x)}\right| \leq K|d|, \text { where } K=\sup _{s \in S}\left|f^{\prime}(x)\right|,
$$

and therefore

$$
-|d| \leq-\frac{1}{K+1}|F(x)| \text { for such }(x, d) .
$$

Utilizing (3.5) this estimate can also be derived for $(x, d)$ which arise as limits of $\left(x^{k}, d^{k}\right)$ in $(A .3)$. Combined with (3.8) this implies that

$$
\max \left(\theta^{o}(x ; d), \theta^{\prime}(x ; d)\right) \leq-\frac{\hat{\sigma}}{(K+1)^{2}}|F(x)|^{2},
$$

for any pair $(x, d)$ satisfying the active set strategy or arising as a limit point in (A.3). Hence the first part of (A.2) and (A.3) hold.

To verify the second part of (A.2), let $(x, d)$ satisfy $G(x ; d)+F(x)=$ $0, x \in S$, and let $\mathcal{A}, \mathcal{I}$ denote the associated active and inactive sets. Then $\left|F(x)_{\mathcal{A}}\right|=\left|d_{\mathcal{A}}\right|$ and $d_{\mathcal{I}}=-A_{\mathcal{I}}^{-1}\left(A_{\mathcal{I}_{\mathcal{A}}} d_{\mathcal{A}}+f(x)_{\mathcal{I}}\right)$. By $(3.7)$

$$
\hat{k}=\sup _{\mathcal{I}, x} A(x)_{\mathcal{I}}^{-1}<\infty
$$

where the sup is taken over all possible partitions into active and inactive sets and $x \in S$. We find that

$$
|d| \leq(\hat{k} K+1)|F(x)|
$$

and hence the second part of (A.2) holds with $b=\hat{k} K+1$. Finally, again by (3.7), the family $\left\{G(x ; \cdot)^{-1}: x \in S\right\}$ is uniformly bounded.

We summarize the findings of this subsection as as a theorem. 
Theorem 3.1. Let $f \in C^{1}$ and assume that (3.7) holds. Then, the direction determined by the primal-dual active set strategy (3.2) satisfies (A.2) and (A.3) with $\theta(x)=|F(x)|^{2}$.

We also note that (A.1) holds for bilateral constraints, and it holds for unilateral constrains, if $\lim _{|x| \rightarrow \infty}|f(x)|=\infty$, for example.

Remark 3.1. If $A=f^{\prime}(x)$ is of the form $A=\beta I+B$ and $\beta$ is sufficiently large compared to $\|B\|$, then (3.7) holds. In fact, there exists $\eta>0$ such that $|B| \leq \eta$ implies that

$$
\begin{aligned}
& \left(A_{33}-A_{32} A_{22}^{-1} A_{23} d, d\right)_{3} \geq \frac{\beta}{2}|d|_{3}^{2} \text { and } \\
& \left|\left(A_{31}-A_{32} A_{22}^{-1} A_{21}\right)^{T}\left(A_{31}-A_{32} A_{22}^{-1} A_{21}\right)+\left(A_{32} A_{22}^{-1}\right)^{T}\left(A_{32} A_{22}^{-1}\right)\right|<\frac{\beta}{2},
\end{aligned}
$$

for any partition $\mathcal{A A}, \mathcal{I} \mathcal{I}, \mathcal{I} \mathcal{A}$. It thus follows that (3.7) holds with $c=1$. The use of $c>0$ enlarges the class of cases for which (3.7) holds. In fact, the critical estimate for positive definiteness requires that an expression of the type $\frac{c \beta}{2}-2 \eta^{2}-\frac{c^{2} \eta^{2}}{\beta^{2}}$ is positive, which is more like to holds for small $c$ than for $c=1$.

Remark 3.2. In optimal control of elliptic equations with pointwise constraints on the controls the necessary optimality condition, after discretization, can be expressed as $F(x)=0$, where

$$
F(x)=f(x)-\max \left(0, f(x)+x-\psi_{h}\right)-\min \left(0, f(x)+x-\phi_{h}\right) .
$$

and

$$
f(x)=\left(\beta I+\Delta_{h}^{-2}\right) x+\Delta_{h}^{-1} z_{h},
$$

where $\Delta_{h}$ denotes the discretized Laplace operator $\Delta, z_{h} \in \mathbb{R}^{m}$ is given, and $\phi_{h}, \psi_{h}$, are the discretized lower and upper bounds, see e.g. [IK].

With reference to condition (3.7) the matrix $A$ is given by $\beta I+\Delta_{h}^{-2}$, and (3.7) is satisfied, if the control cost $\beta$ is sufficiently large. Let also note that due to the fact that $\Delta^{-2}$ is a smoothing operation the matrix norm of $\Delta_{h}^{-2}$ can be considered to be 'small'.

In the following subsection we provide another application for which the primal-dual active direction satisfies (A.2) and (A.3). 


\section{$3.2 \quad \ell^{1}$ - Minimization}

For convenience we recall the definition of $F(x)=f(x)+\frac{x-z}{\max (\epsilon,|x-z|)}$, where $f$ is again assumed to be $C^{1}$. Setting

$$
\begin{aligned}
& \mathcal{A}^{+}=\{x-z>\epsilon\}, \quad \mathcal{A}^{-}=\{x-z<\epsilon\} \\
& \mathcal{I}^{1}=\{x-z=\epsilon\}, \quad \mathcal{I}^{2}=\{-\epsilon<x-z<\epsilon\} \\
& \mathcal{I}^{3}=\{x-z=-\epsilon\}, \quad \mathcal{A}=\mathcal{A}^{+} \cup \mathcal{A}^{-}, \quad \mathcal{I}=\mathcal{I}^{1} \cup \mathcal{I}^{2} \cup \mathcal{I}^{3},
\end{aligned}
$$

we find

$$
F^{\prime}(x ; d)= \begin{cases}f^{\prime}(x) d & \text { on } \mathcal{A}^{+} \cup \mathcal{A}^{-} \\ f^{\prime}(x) d+\frac{d}{\epsilon} \chi_{d<0} & \text { on } \mathcal{I}^{1} \\ f^{\prime}(x) d+\frac{d}{\epsilon} & \text { on } \mathcal{I}^{2} \\ f^{\prime}(x) d+\frac{d}{\epsilon} \chi_{d>0} & \text { on } \mathcal{I}^{3}\end{cases}
$$

where $\chi_{d<0}$ denotes the characteristic function of the set of indices where $d$ is negative. Further

$$
G(x ; d)=\left\{\begin{array}{l}
f^{\prime}(x) d+\frac{d}{\epsilon} \text { on } \mathcal{I}, \\
f^{\prime}(x) \text { on } \mathcal{A} .
\end{array}\right.
$$

The the primal-dual active set directions satisfy

$$
0=G(x) d+F(x)= \begin{cases}f^{\prime}(x) d+f(x)+1 & \text { on } \mathcal{A}^{+} \\ f^{\prime}(x) d+\frac{d}{\epsilon}+f(x)+\frac{x}{\epsilon} & \text { on } \mathcal{I} \\ f^{\prime}(x) d+f(x)-1 & \text { on } \mathcal{A}^{-} .\end{cases}
$$

For $(x, d)$ satisfying $F(x)+G(x ; d)=0$ we have

$$
\frac{1}{2} \theta^{\prime}(x ; d)=-|A d|_{\mathcal{A}}^{2}-\left|A d+\frac{d}{\epsilon}\right|_{\tilde{\mathcal{I}}^{2}}^{2}-\left(A d+\frac{d}{\epsilon}, A d\right)_{\mathcal{I} \backslash \tilde{\mathcal{I}}^{2}},
$$

where $A=f^{\prime}(x)$ and $\tilde{\mathcal{I}}^{2}=\mathcal{I} \cup \tilde{\mathcal{I}}^{1} \cup \tilde{\mathcal{I}}^{3}$, with

$$
\tilde{\mathcal{I}}^{1}=\left\{i \in \mathcal{I}^{1}: d_{i}<0\right\}, \quad \tilde{\mathcal{I}}^{3}=\left\{i \in \mathcal{I}^{3}: d_{i}>0\right\} .
$$

Setting $g=A d$ and $C=I+\frac{1}{\epsilon} A^{-1}$, we have

$$
\frac{1}{2} \theta^{\prime}(x ; g)=-|g|_{\mathcal{A}}^{2}-|C g|_{\tilde{\mathcal{I}}^{2}}-(C g, g)_{\mathcal{I} \backslash \tilde{\mathcal{I}}^{2}},
$$


which is of the same form as (3.4). An analogous expression is is obtained for $\theta^{\circ}(x: g)$ corresponding to (3.6). Proceeding as in Section 3.1 we obtain the following result.

Theorem 3.2. If $f \in C^{1}$ and (3.9) is negative for all $d$, then the steps determined by the primal-dual active set strategy satisfy (A.2) and (A.3) with $\theta(x)=|F(x)|^{2}$. A sufficient condition for (3.9) to be negative is given by (3.7) with $c=1$.

To interpret condition (3.7) for the present situation we consider the case $f(x)=-\Delta_{h}$, where $\Delta_{h}$ denotes the Laplacian discretized by finite elements or finite differences. Then $C=I+\frac{1}{\epsilon}\left(-\Delta_{h}\right)^{-1}$, and hence (3.7) with $A$ replaced by $C$ holds for all $\epsilon$ sufficiently large.

\section{Quasi-Directional Derivative and Descent Directions}

In this section we turn to a discussion of methods for choosing descent directions satisfying (A.2) and (A.3) which may require that a nonlinear system is solved. They can be used in case that the primal-dual active direction fails. As before we let $F: \mathbb{R}^{m} \rightarrow \mathbb{R}^{m}$ be a locally Lipschitz continuous, directionally differentiable function and set $\theta(x)=|F(x)|^{2}$. Further $S$ refers to the set defined in (A.1)

For the Clarke generalized directional derivative $\theta^{0}(x, d)$ we have

$$
\theta^{o}(x, d)=\limsup _{y \rightarrow x, t \rightarrow 0^{+}} \frac{2(F(x), F(y+t d)-F(y))}{t}
$$

and hence there exists $F^{o}: \mathbb{R}^{m} \times \mathbb{R}^{m} \rightarrow \mathbb{R}^{m}$ such that

$$
\theta^{o}(x, d)=2\left(F(x), F^{0}(x, d)\right) \text { for }(x, d) \in \mathbb{R}^{m} \times \mathbb{R}^{m} .
$$

We introduce the notion of quasi-directional derivative.

Definition 4.1. Let $F: \mathbb{R}^{m} \rightarrow \mathbb{R}^{m}$ be directionally differentiable. Then $G: S \times \mathbb{R}^{m} \rightarrow \mathbb{R}^{m}$ is called a quasi-directional derivative of $F$ on $S \subset \mathbb{R}^{m}$ if

(i) $\left(F(x), F^{\prime}(x ; d)\right) \leq(F(x), G(x ; d))$ 
(ii) $G(x ; t d)=t G(x ; d)$, for all $d \in \mathbb{R}^{m}, x \in S$ and $t \geq 0$,

(iii) $\left(F(\bar{x}), F^{o}(\bar{x} ; \bar{d})\right) \leq \limsup _{x \rightarrow \bar{x}, d \rightarrow \bar{d}}(F(x), G(x, d))$ for all $x \rightarrow \bar{x}, d \rightarrow$ $\bar{d}$, with $x, \bar{x} \in S$.

For the special case of optimization subject to box-constraints a quasi-directional derivative will be constructed in Section 4.1. In the remainder of this section we consider the relationship between well-known choices for descent directions, e.g., [HPR, P] and the concept of quasi-directional derivative of Definition 4.1.

(a)Bouligand direction. If there exists $\bar{b}$ such that

$$
|h| \leq \bar{b}\left|F^{\prime}(x ; h)\right| \text { for all } x \in S, h \in \mathbb{R}^{m}
$$

and if

$$
F(x)+F^{\prime}(x ; d)=0,
$$

admits a solution $d$ for each $x \in S$, then a first choice for the direction is given by the solution $d$ to (4.2), see e.g. [P]. By (4.1) we have $|d| \leq \bar{b}|F(x)|$. Moreover

$$
\theta^{\prime}(x, d)=2\left(F^{\prime}(x ; d), F(x)\right)=-2 \theta(x),
$$

and therefore the inequalities in (A.2) hold with $b=\bar{b}$ and $\bar{\sigma}=2$. For this choice, however, (A.3) is not satisfied in general, see Section 4.1. below.

(b) Generalized Bouligand direction. As a second choice, see [HPR, P], we assume that $G$ is a quasi-directional derivative of $F$ on $S$, that

$$
|h| \leq \bar{b}|G(x ; h)| \text { for all } x \in S, h \in \mathbb{R}^{m},
$$

holds and that

$$
F(x)+G(x ; d)=0,
$$

admits a solution $d$ which is used as the descent direction for each $x \in S$. One argues as for the first choice that the inequalities in (A.2) hold with $b=\bar{b}$ and $\bar{\sigma}=2$. Moreover (A.3) is satisfied, since for any $(x, d) \rightarrow(\bar{x}, \bar{d})$ in $S \times \mathbb{R}$ with $d=d(x)$ we have

$$
\theta^{0}(\bar{x}, \bar{d}) \leq 2 \limsup _{x \rightarrow \bar{x}, d \rightarrow \bar{d}}(F(x), G(x ; d))=-2 \lim _{x \rightarrow \bar{x}}|F(x)|^{2}=-2 \theta(\bar{x}) .
$$


We refer to Section 4.1 for the construction of $G$ for specific applications.

(c) Generalized gradient direction. The following choice was discussed in [HPR]. Here $d$ is chosen as the solution to

$$
\min _{d} J(x, d)=2(F(x), G(x ; d))+\eta|d|^{2},
$$

where $\eta>0$ and $x \in S$. Assume that for some $L>0$

$$
\left\{\begin{array}{c}
h \rightarrow G(x ; h) \text { is continuous and } \\
|G(x ; h)| \leq L|h| \text { for all } x \in S, h \in \mathbb{R}^{m} .
\end{array}\right.
$$

Then, $d \rightarrow J(d)$ is coercive, bounded below, and continuous. Thus there exits an optimal solution d to (4.5).

Lemma 4.1. Assume that $F: \mathbb{R}^{m} \rightarrow \mathbb{R}^{m}$ is Lipschitz continuous, directionally differentiable and that $G$ is a quasi-directional derivative of $S$ satisfying (4.6).

(a) If $d$ is an optimal solution to (4.5) then

$$
(F(x), G(x ; d))=-\eta|d|^{2} .
$$

(b) If $d=0$ is an optimal solution to (4.5), then $(F(x), G(x ; h)) \geq 0$ for all $h \in \mathbb{R}^{m}$.

Proof. (a) Let $d$ be an optimal solution to (4.5) and consider

$$
\min _{\alpha \geq 0} 2(F(x), G(x ; \alpha d))+\alpha^{2} \eta|d|^{2} .
$$

Then $\alpha=1$ is optimal and differentiating with respect to $\alpha$ we have

$$
(F(x), G(x ; d))+\eta|d|^{2}=0 .
$$

(b) If $d=0$ is an optimal solution, then the optimal value of (4.5) is zero. It follows that for all $h \in \mathbb{R}^{m}$ and $\alpha \geq 0$

$$
0 \leq 2 \alpha(F(x), G(x ; h))+\alpha^{2} \eta|h|^{2} .
$$

Dividing by $\alpha>0$ and letting $\alpha \rightarrow 0^{+}$we obtain the claim. 
By Lemma 4.1 the optimal value of the cost $J$ in (4.5) is given by $-\eta|d|^{2}$. If this value is negative, then any solution to (4.5) provides a decay for $\theta$ since $\theta^{\prime}(x ; d) \leq-\eta|d|^{2}$. The optimal value of the cost is 0 if and only if $d=0$ is the optimal solution. In this case Lemma 4.1 implies that $x$ is a stationary point in the sense that $(F(x), G(x ; h)) \geq 0$ for all $h \in \mathbb{R}^{m}$.

Let us now turn to the discussion of condition (A.2) for the direction given by the solution $d$ to (4.5). We assume (4.3) and that (4.4) admits a solution for every $x \in S$. Since $J(0)=0$, we have $2(F(x), G(x ; d)) \leq-\eta|d|^{2}$ and therefore

$$
\eta|d|^{2} \leq-2(F(x), G(x ; d)) \leq 2|F(x)||G(x ; d)| \leq 2 L|d||F(x)| .
$$

Thus,

$$
|d| \leq \frac{2 L}{\eta}|F(x)|
$$

and the second condition in (A.2) holds. Turning to the first condition let $\hat{d}$ satisfy $F(x)+G(x ; \hat{d})=0$. Then, using Lemma 4.1(a) and (4.3) we find at a solution $d$ to $(4.5)$

$$
\begin{gathered}
J(x, d)=(F(x), G(x ; d))=-\eta|d|^{2} \leq 2(G(x ; \hat{d}), F(x))+\eta|\hat{d}|^{2} \\
\leq-2|F(x)|^{2}+\eta \bar{b}^{2}|F(x)|^{2}=-\left(2-\eta \bar{b}^{2}\right) \theta(x) .
\end{gathered}
$$

Since $G$ is a quasi-directional derivative of $F$ on $S$ we have $\theta^{\prime}(x ; d) \leq 2(F(x), G(x ; d)) \leq$ $-2\left(2-\eta \bar{b}^{2}\right) \theta(x)$ and thus the direction $d$ defined by (4.5) satisfies the first condition in (A.2) with $\bar{\sigma}=2\left(2-\eta \bar{b}^{2}\right)$, provided that $\eta<\frac{2}{\bar{b}^{2}}$.

To argue (A.3) let $x_{k} \rightarrow \bar{x}, d_{k} \rightarrow \bar{d}$, with $d_{k}=d\left(x_{k}\right), x_{k} \in S$ and choose $\hat{d}_{k}$ such that $F\left(x_{k}\right)+G\left(x_{k} ; \hat{d}_{k}\right)=0$. Then

$$
\begin{aligned}
& \frac{1}{2} \theta^{0}(\bar{x} ; \bar{d}) \leq \limsup _{k \rightarrow \infty}\left(F\left(x_{k}\right), G\left(x_{k} ; d_{k}\right)\right) \\
& \leq \lim \sup _{k \rightarrow \infty}\left(2\left(F\left(x_{k}\right), G\left(x_{k} ; d_{k}\right)\right)+\eta\left|d_{k}\right|^{2}\right) \leq \limsup _{k \rightarrow \infty}\left(2\left(F\left(x_{k}\right), G\left(x_{k} ; \hat{d}_{k}\right)\right)+\eta\left|\hat{d}_{k}\right|^{2}\right) \\
& \leq-2|F(\bar{x})|^{2}+\eta \bar{b}^{2} \lim _{k \rightarrow \infty}\left|F\left(x_{k}\right)\right|^{2}=-\left(2-\eta \bar{b}^{2}\right) \theta(\bar{x}),
\end{aligned}
$$

and thus (A.3) holds if $\eta<\frac{2}{b^{2}}$. 


\subsection{Box Constraints and Quasi-directional Derivative}

Here we show how the above framework applies to the case of bilateral box constraints. The Bouligand direction (4.2) is given by

$$
\begin{aligned}
& d+x-\psi=0 \text { on } \mathcal{A}^{+}, \quad d+x-\phi=0 \text { on } \mathcal{A}^{-}, \quad f^{\prime}(x) d+f(x)=0 \text { on } \mathcal{I}^{2}, \\
& \max \left(f^{\prime}(x) d, c d\right)+F(x)=0 \text { on } \mathcal{I}^{1}, \quad \min \left(f^{\prime}(x) d, c d\right)+F(x)=0 \text { on } \mathcal{I}^{3},
\end{aligned}
$$

and $F^{0}(x, d)$ is given by

$$
F^{o}(x ; d)= \begin{cases}d & \text { on } \mathcal{A}^{+} \cup \mathcal{A}^{-} \\ f^{\prime}(x) d & \text { on } \mathcal{I}^{2} \\ \max \left(f^{\prime}(x) d, c d\right) & \text { on }\{F(x)>0\} \cup\left(\mathcal{I}^{1} \cup \mathcal{I}^{3}\right) \\ \min \left(f^{\prime}(x) d, c d\right) & \text { on }\{F(x) \leq 0\} \cup\left(\mathcal{I}^{1} \cup \mathcal{I}^{3}\right) .\end{cases}
$$

For arbitrary $\delta>0$ we claim that $G$ defined by

$$
G(x ; d)=\left\{\begin{array}{l}
d \text { on } \mathcal{A}_{\delta}=\{-f(x)+c(x-\psi)>\delta\} \cap\{-f(x)+c(x-\phi)<-\delta\}, \\
f^{\prime}(x) d \text { on } \mathcal{I}_{\delta}=\{c(x-\psi)+\delta \leq f(x) \leq c(x-\phi)-\delta\}, \\
\text { and on }\{c \psi-\delta \leq-f(x)+c x<c \psi+\delta\} \cup\{c \phi-\delta \leq-f(x)+c x<c \phi+\delta\}: \\
\max \left(f^{\prime}(x) d, c d\right) \quad \text { if }\{F(x)>0\} \\
\min \left(f^{\prime}(x) d, c d\right) \quad \text { if }\{F(x) \leq 0\},
\end{array}\right.
$$

is a quasi-directional derivative for $F$. In fact, $G$ is clearly positively homogeneous of degree 1 . It is claerly that

$$
\left(F(x), F^{\prime}(x, d)\right) \leq\left(F(x), F^{o}(x, d)\right) \leq(F(x), G(x, d))
$$

which shows (i) of Definition 4.1. If $|x-\bar{x}|$ sufficiently small, then

$$
\begin{aligned}
& i \in \mathcal{I}_{\delta}(x) \text { implies that } i \in \mathcal{I}^{2} \\
& i \in \mathcal{A}_{\delta}(x) \text { implies that } i \in \mathcal{A} .
\end{aligned}
$$

Thus, (iii) holds by the definition of $G$. 


\subsection{Polyhedric Constraint Case}

We now consider (1.2) under the assumption that the closed convex set $\mathcal{C}$ is polyhedric.

Definition 4.2. A closed convex set $\mathcal{C}$ in $\mathbb{R}^{m}$ is called polyhedric at $z \in \mathbb{R}^{m}$, if

$$
\overline{\cup_{\lambda>0} \lambda(\mathcal{C}-P z) \cap[z-P z]^{\perp}}=\overline{\cup_{\lambda>0} \lambda(\mathcal{C}-P z)} \cap[z-P z]^{\perp},
$$

where $P$ denotes the metric projection onto $\mathcal{C}$ and $[z-P z]^{\perp}$ stands for the orthogonal complement of the subspace spanned by $x-P z \in \mathbb{R}^{m}$. Moreover $\mathcal{C}$ is called polyhedric if $\mathcal{C}$ is polyhedric at every $z \in \mathbb{R}^{m}$.

From $[\mathrm{Ha}]$ we have the following result:

Proposition 4.1. Let $\psi$ be an $\mathbb{R}^{m}$-valued function and assume that $\mathcal{C}$ be a closed convex set in $\mathbb{R}^{m}$, which is polyhedric at $\psi(0)$. Set

$$
\hat{\mathcal{K}}(\psi(0))=\overline{\cup_{\lambda>0} \lambda(\mathcal{C}-P \psi(0))} \cap[\psi(0)-P \psi(0)]^{\perp},
$$

and denote by $P_{\hat{\mathcal{K}}(\psi(0))}$ the projection onto $\hat{\mathcal{K}}(\psi(0))$. If there exits a sequence $t_{n}$ such that $\lim _{n \rightarrow \infty} t_{n} \rightarrow 0^{+}$and $\lim _{n \rightarrow \infty} \frac{\psi\left(t_{n}\right)-\psi(0)}{t_{n}}=: \dot{\psi}$ exists in $\mathbb{R}^{m}$, then

$$
\lim _{t_{n} \rightarrow 0^{+}} \frac{P \psi\left(t_{n}\right)-P \psi(0)}{t_{n}}=P_{\hat{\mathcal{K}}(\psi(0))} \dot{\psi}
$$

If $\mathcal{C}$ is polyhedric and $f$ is $C^{1}$, then it follows from Proposition 4.1 that for $F$ defined by (1.2)

$$
F^{\prime}(x ; d)=d-P_{\hat{\mathcal{K}}(x-f(x))}\left(d-f^{\prime}(x) d\right) .
$$

Thus, (4.2) is equivalent to

$$
d+F(x)-P_{\hat{\mathcal{K}}(x-f(x))}\left(d+F(x)-\left(F(x)+f^{\prime}(x) d\right)\right)=0 .
$$

If $f^{\prime}(x) \in \mathbb{R}^{m \times m}$ is symmetric and positive, then (4.2) admits a solution.

In general a quasi-directional derivative $G$ is defined by a quasi-cone $\hat{\mathcal{C}}$ of the cone $\hat{\mathcal{K}}$ at $x-f(x)$ and

$$
G(x, d)=d-P_{\hat{\mathcal{C}}(x-f(x))}\left(d-f^{\prime}(x) d\right) .
$$


Henceforth we examine the case

$$
\mathcal{K}=\{x: B x \leq b\}
$$

where $B: \mathbb{R}^{m} \rightarrow \mathbb{R}^{n}$ is surjective. Defining the slack variable $u=B x-b$, Lagrange multiplier theory allows to express $F$ as

$$
F(x)=f(x)+B^{T} \max (0, B x-b+\mu) \quad \text { with } \quad f(x)+B^{T} \mu=0 .
$$

Thus,

$$
F(x)=f(x)+B^{T} \max \left(0,-\left(B B^{T}\right)^{-1} B f(x)+B x-b\right) .
$$

Setting $\mu=-\left(B B^{T}\right)^{-1} B f(x)$ and using

$F(x)=\left(I-B^{T}\left(B B^{T}\right)^{-1} B\right) f(x)+B^{T}\left(B B^{T}\right)^{-1} B f(x)+B^{T} \max (0, B x-b+\mu)$

, we find

$$
F^{\prime}(x ; d)= \begin{cases}B^{T} B d & \text { on } B^{T}(\{B x-b+\mu>0\}) \\ f^{\prime}(x) d & \text { on } B^{T}(\{B x-b+\mu<0\}) \cup N(B) \\ z(x ; d) & \text { on } B^{T}(\{B x-b+\mu=0\})\end{cases}
$$

where

$$
z(x ; d)=f^{\prime}(x) d+B^{T} \max \left(0,-\left(B B^{T}\right)^{-1} B f^{\prime}(x) d+B d\right),
$$

and we used $\mathbb{R}^{m}=N(B) \oplus R\left(B^{T}\right)$ and $B^{T}\left(B B^{T}\right)^{-1} B=I$ on $R\left(B^{T}\right)$, with $N(B), R\left(B^{T}\right)$ denoting the kernel of $B$, respectively the range of $B^{T}$. Above $F(x ; d)=B^{T} B d$ on $B^{T}(\{B x-b+\mu>0\})$ stands for $F(x ; d)=B^{T} c$, where $c_{i}=(B d)_{i}$ if $(B x-b+\mu)_{i}>0$ and $b_{i}=0$ otherwise. We define the function $G(x ; d)$ by

$$
G(x ; d)=\left\{\begin{array}{l}
B^{T} B d \text { on } B^{T}(\{B x-b+\mu>\delta\}) \\
f^{\prime}(x) d \text { on } B^{T}(\{B x-b+\mu<-\delta\}) \cup N(B), \\
\text { and otherwise: } \\
\max \left(f^{\prime}(x) d, B^{T} B d\right) \text { if }\{F(x)>0\} \\
\min \left(f^{\prime}(x) d, B^{T} B d\right) \text { if }\{F(x) \leq 0\}
\end{array}\right.
$$


With an appropriate coordinate change one can assume that

$$
B^{T} B=I \text { on } R\left(B^{T}\right)
$$

and prove that $G$ is a quasi-directional derivative of $F$.

Acknowledgement: We express our appreciation to the referees and the editors for several suggestions that led to substantial improvements of the paper.

\section{References}

[C] F. H. Clarke: Optimization and Nonsmooth Analysis, John Wiley and Sons, New York, 1983.

[DK] J. C. de los Reyes and K. Kunisch: A comparison of algorithms for control constrained optimal control of the Burgers equation, Calcolo, 41(2004), 203-225.

[FP] F. Facchinei and J.-S. Pang: Finite Dimensional Variational Inequalities and Complementarity Problems vol.II, Springer-Verlag, 2003, Berlin.

[HPR] S-H. Han, J-S. Pang and N. Rangaraj: Globally convergent Newton methods for nonsmooth equations, Mathematics of Operations Research, 17(1992), 586-607.

[Ha] A. Haraux: How to differentiate the projection on a closed convex set in Hilbert space. Some applications to variational inequalities, J. Math. Soc. Japan 29(1977), 615-631.

[HIK] M. Hintermüller, K. Ito and K. Kunisch: The primal-dual active set strategy as a semi-smooth Newton method, SIAM Journal on Optimization 13(2002), 865-888.

[IK] K. Ito and K. Kunisch: The primal-dual active set method for nonlinear optimal control problems with bilateral constraints, SIAM J. on Control and Optimization, 43(2004), 357-376.

[K1] C. Kanzow: Global optimization techniques for mixed complementarity problems, J. Global Optimization 16(2000), 1-21. 
[K2] C. Kanzow: Inexact semismooth Newton methods for large-scale complementarity problems. Optimization Methods and Software 19, (2004), 309-325.

[KR] K. Kunisch and F. Rendl: An Infeasible Active Set Method for Convex Problems with Simple Bounds, SIAM Journal on Optimization, 14(2003), 35-52.

[M] R. Mifflin: Semismooth and semiconvex functions in constrained optimization, SIAM J. Control and Optimization, 15(1977), 959-972.

[OR] J.M. Orthega and W.C. Rheinbold: Iterative Solution of Nonlinear Equations in Several Variables, Acad. Press, New York, 1970.

[P] J.S. Pang: Newton's method for B-differentiable equations, Mathematics of Operations Research, 15(1990), 311-341.

[QS] L. Qi and J. Sun: A nonsmooth version of Newton's method, Math. Programming 58(1993), 353-367.

[Q] L. Qi: Convergence analysis of some algorithms for solving nonsmooth equations, Math. of Operations Research 18(1993), 227-244.

[R] S. M. Robinson: Local structure of feasible sets in nonlinear programming, part III: Stability and sensitivity, Math. Programming Study, 30(1987), 45-66.

[S] A. Shapiro: On concepts of directional differentiability, J. Optim. Theory Appl. 13(1990), 477-487. 